\title{
Kabylie : Cosmogonie
}

\section{Abrous et S. Chaker}

\section{OpenEdition}

Journals

Édition électronique

URL : http://journals.openedition.org/encyclopedieberbere/1443

DOI : 10.4000/encyclopedieberbere.1443

ISSN : 2262-7197

\section{Éditeur}

Peeters Publishers

\section{Édition imprimée}

Date de publication : 1 mai 2004

Pagination : 4086-4093

ISBN : 2-7449-0452-X

ISSN : 1015-7344

\section{Référence électronique}

D. Abrous et S. Chaker, «Kabylie : Cosmogonie », Encyclopédie berbère [En ligne], 26 | 2004, document K20, mis en ligne le 01 juin 2011, consulté le 14 décembre 2020. URL : http://journals.openedition.org/ encyclopedieberbere/1443; DOI : https://doi.org/10.4000/encyclopedieberbere.1443

Ce document a été généré automatiquement le 14 décembre 2020.

(c) Tous droits réservés 


\title{
Kabylie : Cosmogonie
}

\author{
D. Abrous et S. Chaker
}

1 Les travaux ethnographiques et ethnologiques portant sur la Kabylie ont décrit de nombreux rites et pratiques, les qualifiant de « survivances », de «cultes anciens ». Ces rites et pratiques ont survécu, ils persistent alors que la Kabylie est islamisée depuis de nombreux siècles. On peut citer brièvement les rites agraires et les croyances liées au calendrier agricole (anżar, timecreț, amyar n uceqquf, amerdili), les pratiques sacrificielles (asfel), les rites funéraires (asensi), les cultes rendus à différents éléments de la nature (pierres, rochers, sources, arbres); ces éléments, considérés comme le siège du sacré, sont souvent érigés au rang de puissances tutélaires (izessasen) et sont aujourd'hui encore, l'objet d'une véritable dévotion. Ces cultes et pratiques constituent les dernières bribes d'une représentation du monde antérieure à l'Islam, voire même aux trois religions monothéistes. La possibilité de reconstruire ce système de représentation ou au moins certains de ses aspects a toujours buté sur l'inexistence d'un corpus de mythes qui auraient pu éclairer ces rites et pratiques et établir entre eux d'éventuelles relations de cohérence. Henri Basset (1920) a souligné cette absence de mythes dans une formule incisive - «Nul mythe, rien que le rite» - qui s'applique, selon lui, non seulement aux Kabyles mais à l'ensemble des Berbères auxquels il manquerait, pour la production de mythes, «l'indispensable architecte [...] l'imagination qui crée ». Sans atteindre ce constat d'inaptitude prononcé par H. BASSET, une certaine unanimité s'est faite sur l'inexistence de mythes chez les Kabyles et chez les Berbères en général. De rares spécialistes de littérature berbère, à l'instar de P. Galand-Pernet, ont cependant apporté des nuances :

"Il n'y a pas ou peu de mythes berbères comme ceux que l'on connaît dans le domaine indoeuropéen au moins en apparence, affirme-t-elle, [...]. Il est certain que des thèmes se sont échangés entre les différents genres et que l'habillage islamique brouille les pistes; mais même si l'on n'a pas la somme de documents qu'ont explorée les compa-ratistes de l'indoeuropéen ni les repères chronologiques, une meilleure analyse des textes révélerait sans doute des traces de mythes plus importantes qu'on ne le croit et rendrait possibles des reconstructions pour les périodes antéislamiques, y compris préhistoriques». (GalandPernet 1998, p. 112). 
2 Les ethnologues et les spécialistes de littérature orale kabyle, Français dans leur grande majorité, n'ont effectivement pas collecté de mythes; cependant, Leo FroBENIUS, africaniste allemand, a, lors de son séjour en Kabylie au printemps 1914, collecté un corpus de Récits populaires des Kabyles; parmi ces récits publiés en trois volumes entre 1921 et 1922 figure dans le premier volume une série de textes intitulés : " Les mythes de la création et la représentation du monde ". Ces récits collectés par L. Frobenius et publiés en langue allemande n'ont été rendus accessibles aux berbérisants (travaillant dans leur grande majorité en langue française) qu'à la fin des années 1990 : entre 1995 et 1998, paraît la traduction de ces récits de l'allemand vers le français faite par Mokran Fetta et en 1998, paraît le $\mathrm{n}^{\circ} 26$ de Littérature Orale Arabo-Berbère (LOAB) intitulé : «Dossier Leo Frobenius et les «contes kabyles»». Ces deux ouvrages constituent aujourd'hui une référence fondamentale pour réexaminer la question de la production des mythes par les Kabyles.

3 Les récits publiés par L. Frobenius (contes et mythes confondus) posent aux analystes deux épineux problèmes signalés dès 1970 par C. Lacoste-Dujardin dans sa thèse sur Le conte kabyle. Le premier problème est celui de la langue : le texte kabyle de ces récits n'existe pas; ces récits dits en kabyle par des hommes âgés ont été traduits à L. Frobenius en français très probablement par des instituteurs kabyles (Touderti 1998, p. 359) ; ces récits ont ensuite été traduits et publiés en allemand et enfin (re)traduits en français par Mokran FETTA ; ils sont donc passés par quatre étapes : kabyle - français - allemand - français. Sur les conditions de collecte, nous disposons de quelques indices : ces récits ont été contés à FROBENIUS par "des Anciens » (Frobenius cité par Touderti 1998, p. 357-358). Les lieux d'enquête les plus fréquemment cités, y compris dans l'esquisse ethnographique, sont le village d'Aït Bu Mehdi au sud de Draa El Mizan et les Aït-Yanni (Lacoste-Dujardin 1998, p. 54).

4 Face à ce corpus, collecté en 1914 mais nouveau pour les analystes, se pose une question essentielle : celle de sa validation. Cette question a été abordée par trois articles du $\mathrm{n}^{\circ} 26$ de $L O A B$; l'article de Véra Pagin examine, à partir des manuscrits de FROBENIUS, les conditions de collecte et de constitution de ce corpus de « contes kabyles ». L'article de Camille Lacoste-Dujardin porte sur « la validité des contes kabyles de Leo Frobenius » à travers l'exemple du conte de "Ali et sa mère "; quant à l'article de Claude Breteau et Ariette Roth, il est plus précisément consacré à un "essai de validation » de «l'ensemble mythique recueilli par L. Frobenius». Ces trois articles permettent d'affirmer que la question de la validation de ce corpus est loin d'être épuisée ; cependant, et après analyse de la forme et du contenu, la validité des contes et d'une partie de l'ensemble mythique commence à être établie.

5 C'est très précisément ce corpus de mythes qui apporte un éclairage nouveau sur la question de la cosmogonie kabyle. Dans son "esquisse ethnographique " traduite par Ariette Roth (1998, p.153-208), Frobenius consacre une mention spéciale aux précautions qui ont entouré la récitation de ces mythes au moment de leur collecte :

« La découverte [de ces mythes] souligne-t-il, m'a rempli moi-même d'effroi. [...]. Les Leuhdennia, mythes de l'origine du monde sont évidemment un secret très profond et mes vieux conteurs ne cessaient de me supplier de ne jamais les révéler aux Arabes. Il existe différentes règles pour la récitation des mythes. En premier lieu, récitants et auditeurs doivent placer quelques grains de blé sur la langue. La récitation ne peut avoir lieu que la nuit, et jamais au voisinage d'une femme, et, si possible, en dehors de la ferme.

Avant le début de la récitation, lors de la première nuit, on doit sacrifier un coq; à la fin de la quatrième nuit (on ne peut pas les évoquer plus longtemps), on sacrifie 
une chèvre ou un mouton castré de petite taille. Si cette prescription n'est pas respectée, toute la famille du récitant meurt, lui seul demeure en vie ». (Frobenius traduit par Roth 1998, p. 207).

6 Le « pieux effroi » que suscitent ces mythes et toutes les précautions qui entourent leur récitation signifient qu'ils étaient perçus comme un savoir dangereux (pouvant même entraîner la mort) probablement parce qu'il «force» les secrets de l'Invisible. Ces mythes sont eux-mêmes "un secret très profond"; leur apprentissage aurait-il nécessité « une épreuve initiatique » à l'instar de ce que H. Claudot-Hawad décrit pour l'étude de la cosmogonie touarègue ? (Claudot-Hawad 1994, p. 2137) ou alors ces mythes étaient-il maintenus secrets parce que réprouvés par la tradition islamique ? Ce savoir cosmogonique a dû subir face aux trois monothéismes et en particulier à l'Islam un refoulement si profond qu'il a été enterré dans les mémoires. Cette citation appelle une dernière remarque : il s'agit de l'expression Leuh dennia pour dénommer ces mythes. Lamara Bougchiche consacre à cette expression un long développement (Bougchiche 1998, p. 310-312) ; la première lecture donnée par l'auteur est sans doute celle qu'il faut retenir : "lluh $n$ ddenya [...] texte ou livre du monde» (Bougchiche 1998, p. 310). En effet, une des acceptions attestées dans le dictionnaire de Jean-Marie Dallet pour le mot lluḥest bien "planchette de scribe» (Dallet 1982, p. 468); lluḥ n ddenya désignerait donc «Les tablettes du monde». Pour "les vieux conteurs" de FROBENIUS, la référence aux planchettes de l'école coranique (qu'ils devaient sûrement connaître) a dû servir de filtre, permettant d'éluder et d'occulter l'ancienne dénomination car, souligne Frobenius, "ils m'ont prié de leur permettre de garder secret cet ancien nom» (Frobenius traduit par Roth 1998, p. 208).

7 Ce corpus de mythes est aujourd'hui disponible dans deux traductions en langue française : celle de Mokran Fetta (1995, p. 27-96, récits nº 1 à 25$)$ et celle d'Ariette RотH (RоTH 1998: 210-275, récits $\mathrm{n}^{\circ} 1$ à $25 \mathrm{~A}$ ). Ces récits qui ne sont pas tous des mythes cosmogoniques, c'est-à-dire des mythes portant sur l'origine et la formation de l'Univers, présentent une grande diversité thématique ; certains d'entre eux sont plus proches de la légende. Les mythes cosmogoniques identifiés comme tels par C. Breteau et A. Roth (Breteau et Roth 1998, p. 91) comprennent deux séries de récits: une première série dans laquelle émergent des ténèbres souterraines (țțlam) deux couples originels un couple d'humains et un couple de bovines; ce sont les récits $n^{\circ} 1,2,3$ et 4 et une deuxième série dans laquelle l'actant principal est une entité féminine : la Première Mère du Monde ; cette série regroupe les textes n ${ }^{\circ} 5,7,9,10,11,12,14$ et 17.

8 Les autres textes enfin constituent un ensemble hétérogène; ils peuvent porter sur d'autres peuples que les Kabyles (récits n 15, 16, 19, 21), sur des croyances (récits n 22, $23,24)$, etc.

9 Les remarques qui suivent ne portent que sur les deux premières séries. Ce matériau, déjà abordé et validé par le travail de $\mathrm{C}$. Breteau et $\mathrm{A}$. Roth, livre un certain nombre d'éléments entre lesquels il est possible d'établir quelques relations.

10 Un premier point d'ordre général se dégage de ces récits : les éléments qui constituent l'Univers (Terre, Soleil, Lune, Étoiles) et les entités qui peuplent la terre (humains, animaux) ne sont pas présentés comme l'œuvre d'une force créatrice.

11 Plutôt que de création, on pourrait identifier à partir de ces mythes deux processus de formation distincts, il s'agit de :

- l'apparition des êtres humains et de celle des bovinés (récits n 1, 2,3,4); 
- des entités résultant de l'action de la Première Mère du Monde (récits $\mathrm{n}^{\circ} 5,7,9,10,11$, $12,14$ et 17$)$; le récit $n^{\circ} 12$ porte sur la mort de la Première Mère du Monde.

Les humains et les bovinés présentent des ressemblances (récits $n^{\circ} 1$ et 3 ), leur origine est souterraine; ils apparaissent en couples à la surface de la terre; à proximité de l'eau, ils découvrent leur différence sexuelle, s'accouplent et se reproduisent.

- Les humains donnent naissance à cinquante filles et cinquante garçons qui à leur tour s'accouplent (sans savoir que leur union est incestueuse) et peuplent la terre. Un seul couple fait exception : le sauvage et la sauvage (ce sont des humains dénaturés ils n'ont pas construit de maisons et sont devenus anthropophages), ils vécurent dans la forêt ; la sauvage devint la première tteryel (ogresse) et le sauvage le premier lion. De la fourmi, les « premiers parents du monde » apprennent l'agriculture, l'usage du moulin domestique (tissirt $n$ wexxam) et du feu. Ils transmettent ces connaissances aux quarante neuf jeunes couples (récit $n^{\circ} 2$ ).

- La postérité des bovinés, elle, est plus complexe : le buffle originel (izerzer) et la génisse (tawmmatt) s'accouplent et donnent naissance à un taureau; celui-ci sur instruction de la fourmi ( $"$ Vous avez le droit avant les autres animaux de couvrir votre mère et votre sœur »), couvre sa mère alors que celle-ci l'avait repoussé une première fois. Un affrontement s'ensuivit entre le jeune taureau et le buffle-père (Izerzer) qui alla vers les montagnes du Djurdjura près des rochers de Haïzer, y déposa sa semence dans une coupe; de cette semence au contact du soleil, naquirent les animaux sauvages (à l'exception du lion qui provient des humains) qu'Izerzer nourrit et éleva (récit $n^{\circ} 3$ ). De l'union du jeune taureau avec sa mère (après qu'il eût chassé son père Izerzer) naquirent des taureaux et des vaches qui, contraints par le froid (neige) et la faim et se souvenant des conseils de la fourmi, allèrent d'eux-mêmes chez les humains, acceptant une vie confortable bien que plus courte que celle qu'ils auraient menée en liberté (récit $\mathrm{n}^{\circ} 4$ ). Ce processus qui a pour point de départ les humains et les bovinés pourrait être schématisé comme suit :

- humains : accouplement des parents originels $\rightarrow 49$ couples qui construisent des maisons, se multipliant, peuplant la Terre, à l'exception des « sauvages » (Tteryel et le lion).

- bovinés : buffle originel (izerzer) ; génisse $\rightarrow$ taureau.

- taureau - génisse (sa mère) $\rightarrow$ vaches et taureaux domestiques.

- semence du buffle originel. Soleil $\rightarrow$ animaux sauvages (à l'exception du lion d'origine humaine.

$N B$ : La fourmi enseigne aux humains l'agriculture et intervient (par ses conseils) dans la domestication des bovinés (vaches et taureaux).

- Les autres entités qui forment l'Univers, à l'exception de la Terre donnée comme déjà existante, sont l'œuvre de la Première Mère du Monde; elle est à l'origine de certains animaux et des astres les plus «familiers »: le soleil, la lune et les étoiles. La lune et le soleil proviennent eux-mêmes du règne animal, des animaux domestiques (bœuf et mouton).

- Les animaux domestiques ont été « crées " par modelage : avec de la farine qu'elle a obtenue en moulant du grain, la Première Mère du Monde façonne une brebis puis un bélier puis d'autres moutons, elle les nourrit de couscous. La fourmi renseigne les humains sur l'utilité des moutons (viande, laine) et sur le rôle dans la célébration des grandes fêtes: sont citées les fêtes musulmanes (lkid tamez̧̧yant, leid tameqqrant, tacacurt, lmulud) en relation avec les mois du calendrier julien. 
13 Le premier bélier façonné ne mourut pas comme les autres animaux : « il monta un jour très haut dans les montagnes, si haut qu'il cogna avec sa tête contre le soleil montant [à l'horizon]. Le soleil se fixa sur lui et, depuis, il [le bélier] se déplace de conserve avec lui [le soleil] ». (Frobenius, traduction A. Roth 1998, p. 229-230 / récit nº 5).

- D’autres animaux résultent de la métamorphose sur mauvais conseils de la Première Mère de Monde ou dans un de ses accès de colère; c'est le cas d'un jeune garçon transformé en singe (récit $\left.n^{\circ} 10\right)$, du hérisson « qui était à l'origine constitué comme un être humain de petite taille » (idem, 1998 : 237), du porc-épic qui était un jeune mouton et de la tortue qui était un taurillon (récit $n^{\circ} 11$ ). La Première Mère du Monde n'intervient pas dans l'apparition de la puce et du chien (récit $n^{\circ} 6$ ).

- Les astres (la lune et le soleil) résultent des soins que la Première Mère du Monde apporte aux yeux du bœuf (azger) et du mouton (izimmer). La partie tuméfiée de la paupière du bœuf jetée dans l'eau donne la lune, La partie tuméfiée de la paupière du mouton jetée dans le feu donne le soleil (récit $n^{\circ} 14$ ).

14 La première éclipse du soleil résulte d'un acte de sorcellerie : la Première Mère du Monde fit tomber le soleil dans un grand plat en bois et consentit, pour ceci, le premier sacrifice humain (récit $\left.n^{\circ} 7\right)$.

a Première Mère du Monde est à l'origine de la mort des humains en raison de mauvais conseils qu'elle aurait donnés à une jeune femme (récit $\left.n^{\circ} 17\right)$. Dieu intervient dans ce récit.

16 Après tous les méfaits qu'elle commit, la Première Mère du Monde mourut pétrifiée de froid avec ses moutons et ses bœufs lors des gelées de Janvier (Yennayer) (récit $\mathrm{n}^{\circ} 12$ ).

Il s'agit là, exposés brièvement, des éléments essentiels fournis par ce corpus sur l'origine de l'Univers et des entités qui peuplent la Terre. Le processus de formation de toutes ces entités n'est pas linéaire, il présente un réseau de ramifications dont le point de départ se trouve dans les ténèbres souterraines. Outre la production sexuée dont sont issus les humains, le taureau et les bovins domestiques, le soleil ou la chaleur ont permis la génération des animaux sauvages (à partir de la semence d'Izerzer), de la puce et du chien (pour la chaleur du fumier [récit $\left.n^{\circ} 6\right]$ ).

On notera enfin la présence des éléments (l'eau et le feu) associés à des substances animales (paupières de bœuf et de mouton) dans la formation de la lune et du soleil.

Les trois principaux actants de cette genèse - le buffle originel, la Première Mère du Monde et la fourmi - sont des figures archaïques; le buffle originel n'intervient que dans les processus de génération, les rôles les plus importants reviennent à la Première Mère du Monde et à la fourmi, cette dernière a une fonction civilisatrice (enseigne l'agriculture, intervient dans la domestication des bovins et des ovins qui, en réalité sont « nés » domestiques).

20 Tous ces éléments indiquent une représentation de l'origine $\mathrm{du}$ monde très probablement antérieure au monothéisme; C. Breteau et A. Roth pensent « qu’il pourrait s'agir d'un mythe polythéiste afro-méditerranéen, de caractère agraire susceptible d'avoir été réaffecté, à des fins idéologiques qui restent difficiles à déterminer.» (Breteau - Roth 1998, p. 118).

21 L'incompatibilité de ces mythes avec la vision monothéiste de l'origine du monde explique le profond refoulement dont ils font l'objet; il ne s'agit cependant pas d'une amnésie totale: certains récits (exemple: celui relatif à l'origine du singe et de la 
tortue) sont encore connus et de nombreux thèmes et motifs compris dans ces récits sont encore attestés.

À «L'inventaire provisoire des thèmes et motifs » ouvert par C. Breteau et A. Roth (Breteau - Roth 1998, p. 134-141), une première exploration portant sur des données kabyles permet d'apporter d'autres éléments; ces éléments relèvent du domaine des croyances, de celui de la littérature orale et de celui de la langue ; la liste reste, bien sûr, ouverte.

Dans le domaine des croyances :

- Les derniers jours de Janvier et les gelées qui ont fait périr la Vieille et ses animaux (amerdilil) sont encore identifiés dans le calendrier agricole.

- La pratique de sorcellerie qui consiste à faire «tomber la lune » dans un plat en bois est encore perçue comme redoutable car elle exige le sacrifice d'un être cher.

- Le thème du taureau porteur de l'Univers est encore associé aux tremblements de terre, (ddunit yef yiccew $n$ wezger : le monde [repose] sur la corne du boeuf).

Dans le domaine de la littérature orale :

- Une légende présente une sainte anachorète qui a donné son nom au point culminant du Djurdjura (Yemma Xliğa Tukrift : Mère Khlidja la Percluse) sous des traits semblables à ceux de la Première Mère du Monde. «Dans son ermitage de Tamgout »; son don de voyance est attribué à " deux chevreaux qu'elle possédait »; elle est décrite "en train de tourner la meule de son moulin à bras » (Mammeri 1980, p. 381).

Dans le domaine de la langue :

- l'expression : segg wasmi tella yemma-s $n$ ddunit : « depuis qu'existait la Première Mère du Monde » fait référence à un passé très lointain, aux temps les plus reculés.

- Itri : l'étoile ; ce mot (itri) désigne aussi toute tache blanche visible sur l'œil.

- le nom du buffle originel (izerzer), qui désigne également la gazelle, est conservé dans une expression: Aniwer la messdent tferyin, yer Eli Izerzer meskin : "vers qui (contre qui) les coutelas sont-ils aiguisés contre le pauvre Ali Izerzer ». Se dit d'une punition, d'un danger auquel on s'attend.

Il faut souligner enfin que certains des thèmes et motifs compris dans ces mythes sont présents dans la littérature kabyle moderne; c'est le cas notamment pour deux écrivains : Rachid Aliche et Amar Mezdad. Dans le dernier recueil de nouvelles publié par A. Mezdad (Mezdad 2003, p. 123-139), une nouvelle entièrement écrite en kabyle et traduite en français : Eux, le corbeau et nous est construite sur la trame du récit $n^{\circ} 16 \mathrm{du}$ corpus de Frobenius «le message de Dieu et les dons aux peuples", l'actant principal y est un corbeau (tagerfa).

27 Ces mythes, bien que refoulés, affleurent, empruntant plusieurs voies. Ils constituent aujourd'hui un champ de recherche à peine exploré qui pourrait ouvrir sur de riches perspectives.

\section{NOTE COMPLÉMENTAIRE (S. Chaker)}

Il n'existe qu'une seule tentative de synthèse et d'inventaire systématique des croyances et pratiques religieuses des Berbères, à travers les lieux et les temps, celle de Werner Vycichl : «Die Mythologie der Berber », parue dans le Wörterbuch der Mythologie (H.-W. Haussig, ed.), Stuttgart, Ernst Klett Verlag, 1972, p. 555-708. 

raison de la langue; il est cependant le seul à avoir exploité méthodiquement les différentes sources disponibles :

Sources archéologiques et historiques égyptiennes ;

Sources littéraires grecques et latines ;

Sources arabes ;

Sources espagnoles relatives à la population indigène (berbère) des Canaries; Matériaux archéologiques divers, funéraires et art rupestre; Matériaux ethnographiques (littéraires et autres) relatifs au monde berbère contemporain.

On notera qu'il a été le premier à avoir intégré les matériaux très importants et originaux de Leo Frobenius sur la Kabylie.

On dispose là d'une base de départ sérieuse pour une reconstitution globale du système des croyances des Berbères avant l'Islam, perspective qui suppose évidemment la collaboration de l'historien, de l'ethnologue et du spécialiste des religions, ainsi qu'un travail considérable d'exploration et/ou de relecture de nombreuses sources, notamment ethnographiques.

L'analyse sémiologique des représentations rupestres est sans doute l'autre piste principale pour une reconstruction de la cosmogonie berbère; des travaux récents comme celui de S. Hachi (1998) confirment la possibilité d'une mise en relation assez directe - et convaincante - entre ces représentations rupestres préhistoriques et les croyances et représentations symboliques et mythologiques actuelles de certains groupes berbères.

\section{BIBLIOGRAPHIE}

\section{(D. Abrous)}

Basset H., 1920, Essai sur la littérature des Berbères, Editions Carbonnel, Alger.

Bougchiche L., 1998, «Glossaire kabyle des termes et des énoncés figurant dans les chapitres liminaires et dans l'ensemble mythique du volume I des Volksmärchen der Kabylen (p. 3-114)», Littérature Orale Arabo-Berbère, 26 : Dossier Leo Frobenius et les « contes kabyles » (p. 277-338), Paris, Éditions du Cnrs.

Bourdieu P., 1972, Esquisse d'une théorie de la pratique. Précédé de trois études d'ethnologie kabyle, Librairie Droz, Genève (Suisse).

Bourdieu P., 1980, Le sens pratique, Editions de Minuit, Paris.

Breteau Cl. H., Roth A., 1998, « L'ensemble mythique recueilli par Leo Frobenius. Un essai de validation », Littérature Orale Arabo-Berbère, 26 : Dossier Leo Frobenius et les « contes kabyles » (p. 75-148), Paris, Éditions du Cnrs.

Camps G., 1989, « Animisme », Encyclopédie Berbère V (p. 660-672), Aix-en-Provence, Edisud.

Camps G, 1989, « Anz̦ar », Encyclopédie Berbère, VI (p. 795-797), Aix-en-Provence, Edisud. 
Chaker S., 1989, « AnZ̦ar », Encyclopédie Berbère, VI (p. 798), Aix-en-Provence, Edisud.

Chaker S. \& Claudot-Hawad H., 1989, « Arc-en-ciel », Encyclopédie Berbère, VI (p. 861-862), Aix-enProvence, Edisud.

Champault D., 1989, « Arbres sacrés », Encyclopédie Berbère, VI, Aix-en-Provence, Edisud.

Claudot-Hawad H., 1994, « Cosmogonie touarègue », Encyclopédie Berbère, XIV, Aix-en-Provence, Edisud.

Dallet J-M., 1959, « Iressasen, agraw lleywat. Les Gardiens, l'assemblée. Eléments de mystagogie kabyle », Fichier de Documentation Berbère (Fdb), $n^{\circ} 63$, Fort-National.

Fetta M., 1995-1998, Contes kabyles, recueillis par Leo Frobenius. Volumes I, 2, 3 et 4 (Préface de Camille Lacoste-Dujardin). Edisud, Aix-en-Provence.

Fichier de Documentation Berbère $(F d b)$ n 74, 1962 - « La mort, le deuil, les rites funèbres ».

Galand-Pernet P., 1998, Littérature berbères - Des voix. Des lettres. Presses Universitaires de France, Paris.

Genevoix H., 1975 (1), « Le calendrier agraire et sa composition », Le Fichier Périodique, 125, Alger.

Genevois H., 1994, « Croyances », Encyclopédie Berbère, XIV, Aix-en-Provence, Edisud.

Hachi S., 1998, « Une approche anthropologique de l'art figuratif préhistorique d'Afrique du Nord », Études et Documents Berbères, 15-16, p. 163-184 (2000).

Hachid M., 2000, Les Premiers Berbères entre Méditerranée, Tassili et Nil, (en particulier pages 229 à 268). Ina-yas, Alger - Edisud, Aix-en-Provence.

Lacoste-Dujardin C, 1969, Le conte kabyle : Etude ethnologique, Maspéro, Paris.

Lacoste-Dujardin C, 1981, " Maghreb. Éléments de mythologie kabyle », in Y. Bonnefoy (éd). Dictionnaire des mythologies et des religions des sociétés traditionnelles et du monde antique, II. Ed. Flammarion, Paris.

Lacoste-Dujardin C, 1998, La validité des contes kabyles de Leo Frobenius. Exemple de « Ali et sa mère ", Littérature Orale Arabo-Berbère, 26 : Dossier Leo Frobenius et les « contes kabyles » (p. 51-74), Paris, Éditions du Cnrs.

Laoust-Chantréaux G., 1990, Kabylie côté femmes. La vie féminine à Aït-Hichem 1937-1939, Aix-enProvence, Edisud.

Mammeri M., 1980, Poèmes kabyles anciens, Editions Maspéro, Paris.

Pagin V, 1998, « Leo Frobenius, un ethnologue à réévaluer », Littérature Orale Arabo-Berbère, 26: Dossier Leo Frobenius et les « contes kabyles » (p. 7-50), Paris, Editions du Cnrs.

Servier J., 1962, Les portes de l'année, Editions Laffont, Paris. Réédité en 1985 sous le titre : Traditions et Civilisations berbères, Editions du Rocher, Monaco.

Touderti A., 1998, « De l'écritoire à l'équateur, le séjour de Leo Frobenius en Kabylie et les conditions de recueil des contes ", Littérature Orale Arabo-Berbère, 26 : Dossier Leo Frobenius et les « contes kabyles» (p. 355-360), Paris, Éditions du Cnrs.

Vycichl W. : « Die Mythologie der Berber », in Wörterbuch der Mythologie (H.-W. Haussig, ed.), Stuttgart, Ernst Klett Verlag, 1972, p. 555-708. 
INDEX

Mots-clés : Cosmogonie, Croyances, Ethnologie, Kabylie 